Bundesgesundheitsbl 2022 $\cdot 65: 40-46$ https://doi.org/10.1007/s00103-021-03466-y Eingegangen: 20. Juni 2021

Angenommen: 15. November 2021

Online publiziert: 8. Dezember 2021

(c) Der/die Autor(en) 2021

Tobias Teismann' · Anja Gysin-Maillart ${ }^{2,3,4}$

'Forschungs- und Behandlungszentrum für Psychische Gesundheit, Fakultät für Psychologie, RuhrUniversität Bochum, Bochum, Deutschland

${ }^{2}$ Translationales Forschungszentrum, Universitätsklinik für Psychiatrie und Psychotherapie Bern, Universität Bern, Bern, Schweiz

${ }^{3}$ Abteilung für Medizinische Psychologie und Medizinische Soziologie, Universität Leipzig, Leipzig, Deutschland

${ }^{4}$ Abteilung klinische Suizidforschung, Departement für klinische Forschung und Psychiatrie, Medizinische Fakultät, Universität Lund, Lund, Schweden

\title{
Psychotherapie nach einem Suizidversuch - Evidenzlage und Bewertung
}

such entwickelt und evaluiert wurden. Hierzu werden Befunde aus Metaanalysen - insbesondere 2 aktuelle CochraneReviews - zusammengefasst und Beispiele für effektive Behandlungsansätze gegeben. Es folgt eine kritische Würdigung der gegenwärtigen Befundlage.

\section{Evidenzen aus systematischen Reviews und Metaanalysen} zidversuche gelten - neben psychischen Erkrankungen - als zentraler Risikofaktor für Suizide [4]. In einer Metaanalyse kommen Caroll et al. [5] zu dem Ergebnis, dass das Risiko, durch einen Suizid $\mathrm{zu}$ versterben, im ersten Jahr nach einem Suizidversuch bei 1,6\%, nach 5 Jahren bei $3,9 \%$ und nach 10 Jahren bei $4,2 \%$ liegt. Das Risiko eines erneuten Suizidversuchs wird im ersten Jahr auf $16,3 \%$ und binnen der ersten 5 Folgejahre auf 22,4\% geschätzt [5]. Bei den allermeisten Betroffenen bleibt ein Suizidversuch somit ein singuläres Ereignis in ihrem Leben; gleichzeitig ist das Risiko, an einem Suizid zu sterben, nie so hoch wie im ersten Jahr nach einem Suizidversuch. Entsprechend stellt sich die Frage, mit welchen psychotherapeutischen Maßnahmen dem erhöhten Wiederholungs- und Mortalitätsrisiko begegnet werden kann.

Ziel dieser Arbeit ist es, einen narrativen Überblick über Psychotherapieverfahren zu geben, die speziell zur Behandlung von Personen nach Suizidver-
Im Rahmen von 2 aktuellen CochraneReviews haben Witt et al. [6, 7] Studien zur Effektivität psychosozialer Interventionen bei der Behandlung von Erwachsenen und von Kindern/Jugendlichen zusammengefasst. Einbezogen in die Metaanalysen wurden ausschließlich randomisiert kontrollierte Studien, in denen eine psychosoziale Intervention mit einer Vergleichsbedingung (zumeist Regelversorgung, engl. ,treatment as usual“, TAU) verglichen wurde. Ein weiteres Einschlusskriterium sah vor, dass sich alle Patient ${ }^{*}$ innen innerhalb der letzten 6 Monate vor Studieneinschluss absichtsvoll selbstverletzt (engl. „self-harm“) haben mussten. Primäres Outcome-Maß war das erneute Auftreten intentionaler Selbstverletzung. Insgesamt wurden 76 Studien $(N=21.414)$ mit Fokus auf erwachsene Patient ${ }^{*}$ innen [6] und 17 Stu$\operatorname{dien}(N=2280)$ mit Fokus auf Kinder und Jugendliche [7] identifiziert.

Bei einer weitgehend unzureichenden Studiengüte fanden sich bei Erwachse- nen Hinweise darauf, dass kognitive Verhaltenstherapie (KVT) 6 (OR 0,52, $95 \%$ Konfidenzintervall (KI) 0,38-0,70; $n=1260$; Anzahl der Studien $(k)=12$ ) und 12 Monate (OR 0,81, $95 \%$ KI 0,66-0,99; $n=2458 ; k=9)$ nach Therapieende $\mathrm{zu}$ einer Reduktion erneuter Selbstverletzungen beiträgt [6]. Zudem zeigte sich eine Reduktion von Suizidgedanken zum Therapieende und 6 Monate nach Therapieende (siehe auch [8]). In Bezug auf die dialektisch-behaviorale Therapie (DBT) fanden Witt et al. [6] keinen Effekt im Hinblick auf das Auftreten von selbstverletzendem Verhalten, wohl aber mit Blick auf die Häufigkeit, mit der erwachsene Patient*innen selbstverletzendes Verhalten zu Therapieende zeigten (Mean Difference (MD) $-5,00$, $95 \%$ KI $-8,92$ bis $-1,08 ; n=659 ; k=7$ ). In Bezug auf unterschiedliche DBTGestaltungsformen fanden sich keine differenziellen Befunde und es zeigte sich kein Behandlungseffekt hinsichtlich Suizidgedanken. In weiteren Studien erwiesen sich die mentalisierungsbasierte Therapie (MBT) und eine auf Emotionsregulation fokussierende Gruppentherapie als effektiv hinsichtlich einer Reduktion selbstverletzenden Verhaltens.

Im Kindes- und Jugendalter zeigten sich positive Effekte ausschließlich für die dialektisch-behaviorale Therapie (DBTA) zum Postzeitpunkt (OR 0,46, $95 \% \mathrm{KI}$ $0,26-0,82 ; n=270 ; k=4)$; weder in Bezug 


\begin{tabular}{|c|c|c|c|c|c|c|}
\hline Autor*innen & Jahr & $\begin{array}{l}\text { Therapiemethode im Fo- } \\
\text { kus }\end{array}$ & $\begin{array}{l}\text { Anzahl } \\
\text { Studien } \\
(k)\end{array}$ & $\begin{array}{l}\text { Anzahl Pro- } \\
\text { band*innen } \\
(N)\end{array}$ & $\begin{array}{l}\text { Primäres Out- } \\
\text { come-Maß }\end{array}$ & Ergebnis \\
\hline $\begin{array}{l}\text { Bornheimer } \\
\text { et al. [18] }\end{array}$ & 2020 & $\begin{array}{l}\text { Psychosoziale Interventio- } \\
\text { nen bei Psychosen }\end{array}$ & 11 & 4829 & $\begin{array}{l}\text { Suizidgedanken, } \\
\text { Suizidversuch, } \\
\text { Suizid }\end{array}$ & $\begin{array}{l}\text { BG > KG bzgl. Suizidgedanken } \\
(\mathrm{OR}=0,73,95 \% \mathrm{KI}, 0,55-0,97) \\
\text { BG > KG bzgl. Suizide } \\
(\mathrm{OR}=0,45,95 \% \mathrm{KI} 0,30-0,68) \\
\text { BG }=\text { KG bzgl. Suizidversuche }\end{array}$ \\
\hline $\begin{array}{l}\text { Briggs et al. } \\
{[19]}\end{array}$ & 2019 & $\begin{array}{l}\text { Psychoanalyse/ } \\
\text { Psychodynamische Therapie } \\
\text { (PA/PD) }\end{array}$ & 12 & 999 & $\begin{array}{l}\text { Suizidversuch, } \\
\text { "self-harm" }\end{array}$ & $\begin{array}{l}\text { PA/PD > KG bzgl. Suizidversuche } \\
\text { (OR = 0,47, } 95 \% \text { KI 0,27-0,80) } \\
\text { PA/PD > KG bzgl. „self-harm“ (6 Monate FU: } \\
\text { OR=0,27, } 95 \% \text { KI 0,10-0,66) } \\
\text { PA/PD = KG bzgl. Anzahl Suizidversuche, Anzahl } \\
\text { Self-harm-Episoden, „self-harm“ (12 Monate FU) }\end{array}$ \\
\hline $\begin{array}{l}\text { Büscher et al. } \\
\text { [20] }\end{array}$ & 2020 & $\begin{array}{l}\text { Internetbasierte kognitive } \\
\text { Verhaltenstherapie (KVT) }\end{array}$ & 6 & 1567 & Suizidgedanken & $\begin{array}{l}\text { KVT > KG bzgl. Suizidgedanken (SMD }=-0,29 \text {; } \\
95 \% \mathrm{KI}-0,40 \text { bis }-0,19)\end{array}$ \\
\hline $\begin{array}{l}\text { Calati und } \\
\text { Courtet [21] }\end{array}$ & 2016 & Psychotherapie & 32 & 4114 & $\begin{array}{l}\text { Suizidversuch, } \\
\text { NSSI }\end{array}$ & $\begin{array}{l}\text { BG > KG bzgl. Suizidversuche } \\
\text { (Suizidversuchsrate: } 9,12 \% \text { vs. } 15,71 \% \text { ) } \\
\text { BG = KG bzgl. NSSI }\end{array}$ \\
\hline $\begin{array}{l}\text { DeCou et al. } \\
{[22]}\end{array}$ & 2019 & $\begin{array}{l}\text { Dialektisch-behaviorale } \\
\text { Therapie (DBT) }\end{array}$ & 18 & 987 & Self-harm & $\begin{array}{l}\text { DBT > KG bzgl. „self-harm“ } \\
(d=-0,32,95 \% \mathrm{KI}-0,47 \text { bis }-0,17) \\
\text { DBT }=\text { KG bzgl. Suizidgedanken }\end{array}$ \\
\hline $\begin{array}{l}\text { Fox et al. } \\
\text { [23] }\end{array}$ & 2020 & $\begin{array}{l}\text { Psychosoziale Interven- } \\
\text { tionen, Psychotherapie, } \\
\text { Pharmakotherapie }\end{array}$ & 1125 & - & $\begin{array}{l}\text { Suizidgedanken, } \\
\text { Suizidversuche, } \\
\text { „self-harm“, NSSI, } \\
\text { Suizid }\end{array}$ & $\begin{array}{l}\text { BG }>\text { KG bzgl. Suizidgedanken } \\
(g=-0,09 ; 95 \% \mathrm{KI}-0,15 \text { bis }-0,02) \\
\text { BG }=\text { KG bzgl. Suizidversuche, Suizid, NSSI, „self- } \\
\text { harm“ (jeweils Posttreatment) }\end{array}$ \\
\hline $\begin{array}{l}\text { Kothgassner } \\
\text { et al. [24] }\end{array}$ & 2020 & $\begin{array}{l}\text { Psychotherapie bei Jugendli- } \\
\text { chen }\end{array}$ & 25 & 2962 & $\begin{array}{l}\text { Self-harm, Suizid- } \\
\text { gedanken }\end{array}$ & $\begin{array}{l}\text { BG > KG bzgl. „self-harm“ } \\
(d=0,13,95 \% \text { KI 0,04-0,22) } \\
\text { BG }>\text { KG bzgl. Suizidgedanken } \\
(d=0,31,95 \% \text { KI 0,12-0,50) }\end{array}$ \\
\hline $\begin{array}{l}\text { Leavey und } \\
\text { Hawkins [25] }\end{array}$ & 2017 & $\begin{array}{l}\text { Kognitive Verhaltensthera- } \\
\text { pie }\end{array}$ & 26 & 2790 & $\begin{array}{l}\text { Suizidalität (Sui- } \\
\text { zidgedanken, } \\
\text { Suizidversuche) }\end{array}$ & $\begin{array}{l}\text { BG (Face-to-Face-Behandlung) }>\text { KG bzgl. Suizida- } \\
\text { lität } \\
(\mathrm{SMD}=-0,24,95 \% \mathrm{KI}-0,41 \text { bis }-0,07)\end{array}$ \\
\hline $\begin{array}{l}\text { Meerwjik et al. } \\
{[26]}\end{array}$ & 2016 & $\begin{array}{l}\text { Spezifische vs. unspezifi- } \\
\text { sche psychosoziale und } \\
\text { psychotherapeutische Inter- } \\
\text { ventionen }\end{array}$ & 44 & $\begin{array}{l}13.369 \text { (Post- } \\
\text { messung) } \\
11.887 \text { (FU- } \\
\text { Messung) }\end{array}$ & Suizidversuche & $\begin{array}{l}\text { Direkte Intervention }=\text { indirekte Intervention } \\
\text { zum Postzeitpunkt (OR=0,62 vs. } 0,93, t=-2,04, \\
p=0,06 ; d=0,77) \\
\text { Direkte Intervention }=\text { indirekte Intervention } \\
\text { zum FU-Zeitpunkt (OR=0,65 vs. } 0,82, t=-1,20, \\
p=0,25 ; d=0,47)\end{array}$ \\
\hline $\begin{array}{l}\text { Padmanathan } \\
\text { et al. [27] }\end{array}$ & 2020 & $\begin{array}{l}\text { Psychosoziale Interventio- } \\
\text { nen/Pharmakotherapie bei } \\
\text { Substanzkonsumstörungen }\end{array}$ & 6 & 468 & $\begin{array}{l}\text { Suizidalität (Sui- } \\
\text { zidgedanken, } \\
\text { „self-harm") }\end{array}$ & $\begin{array}{l}\text { BG }>\text { KG bzgl. Suizidalität } \\
(d=-0,20,95 \% \mathrm{KI}-0,39 \text { bis }-0,00)\end{array}$ \\
\hline $\begin{array}{l}\text { Riblet et al. } \\
{[28]}\end{array}$ & 2017 & $\begin{array}{l}\text { Psychosoziale Interven- } \\
\text { tionen, Psychotherapie, } \\
\text { Pharmakotherapie }\end{array}$ & 24 & 3056 & Suizid & $\begin{array}{l}\text { KVT }=\text { KG bzgl. Suizid } \\
(\mathrm{OR}=0,34,95 \% \mathrm{KI} 0,12-1,03)\end{array}$ \\
\hline $\begin{array}{l}\text { Swift et al. } \\
{[29]}\end{array}$ & 2021 & $\begin{array}{l}\text { Collaborative Assessment } \\
\text { and Management of Suicida- } \\
\text { lity (CAMS) }\end{array}$ & 9 & 749 & Suizidgedanken & $\begin{array}{l}\text { CAMS > KG bzgl. Suizidgedanken } \\
(d=0,25,95 \% \text { KI 0,07-0,42) } \\
\text { CAMS = KG bzgl. Suizidversuche, „self-harm“ }\end{array}$ \\
\hline $\begin{array}{l}\text { Torok et al. } \\
\text { [30] }\end{array}$ & 2020 & Internetbasierte Therapie & 16 & 4398 & Suizidgedanken & $\begin{array}{l}\text { BG > KG bzgl. Suizidgedanken } \\
\text { (Hedges } g=-0,18,95 \% \mathrm{KI}-0,27 \text { bis }-0,10 \text { ) }\end{array}$ \\
\hline
\end{tabular}

auf KVT noch MBT fanden sich entsprechende Wirksamkeitsnachweise [7].

Kritisch lässt sich anmerken, dass in beiden Cochrane-Reviews - im Sinne der englischen Tradition [9] - nicht zwischen Selbstverletzungen in suizidaler und nichtsuizidaler Absicht differenziert wird. Es bleibt daher unklar, ob und inwieweit die Befunde gleichermaßen für nichtsuizidale Selbstverletzungen wie für Suizidversuche gelten. Vor diesem Hintergrund wurde von Gøtzsche und Gøtzsche [10] eine Reanalyse auf Basis einer älteren Fassung des Cochrane-Reviews [11] zur psychosozialen Suizidprävention im Erwachsenenalter vorgenommen. In die Metaanalyse wurden 10 randomisiert kontrollier- 
te Studien eingeschlossen, in denen kognitive Verhaltenstherapie mit TAU verglichen wurde. Primäres OutcomeMaß waren erneute Suizidversuche (im Unterschied zu einem integrierten Maß „self-harm“). Es zeigte sich eine nahezu 50 \%ige Reduktion des Risikos eines erneuten Suizidversuchs infolge einer KVT im Vergleich zu einer TAU-Behandlung (Risk Ratio 0,47, 95\%, KI $0,30-0,73 ; N=1241$ ). Die Befunde verweisen zum einen auf die Effektivität (einzelner) KVT-Behandlungsprogramme (siehe unten) und zum anderen auf die Bedeutung, zwischen suizidaler und nichtsuizidaler Selbstverletzung als Outcome-Maß zu differenzieren.

In weiteren Metaanalysen wurde der Effekt psychotherapeutischer Suizidprävention untersucht, ohne dass vorangegangenes selbstverletzendes Verhalten als Einschlusskriterium gefordert wurde. Tab. 1 gibt einen Überblick über entsprechende Metaanalysen, die in den Jahren 2016-2021 publiziert wurden. Dabei wurden Metaanalysen, die unkontrollierte Prä-Post-Designs [12-14] inkludieren, genauso wenig berücksichtigt wie eine Metaanalyse zu Kurz- und Kontaktinterventionen [15], eine $\mathrm{Me}$ taanalyse zum Vergleich verschiedener TAU-Bedingungen [16] und eine Metaanalyse zu Behandlungsabbrüchen [17].

Alles in allem findet sich in Metaanalysen - mit variierenden Einschlusskriterien - ein präventiver Effekt psychotherapeutischer Interventionen auf die Rate von Patient*innen, die erneute Suizidversuche und/oder Selbstverletzungen vornehmen, sowie auf die Reduktion von Suizidgedanken. Ein präventiver Effekt auf die Anzahl an Suiziden findet sich nur in einer einzelnen Studie [27]. Grundsätzlich scheinen Verfahren, die suizidales Erleben und Verhalten zum expliziten Fokus der Behandlung machen, effektiver zu sein als Verfahren, die auf die Reduktion assoziierter Psychopathologie (z. B. Depression, Hoffnungslosigkeit) abzielen [26, 30], allerdings braucht es diesbezüglich noch weitere Bestätigung [23]. Einschränkend ist schlussendlich hervorzuheben, dass (1) die Effekte zumeist nur von geringer Größe sind, (2) sich - in Abhängigkeit von den Einschlusskriterien, Outcome-Variablen

Bundesgesundheitsbl 2022 -65:40-46 https://doi.org/10.1007/s00103-021-03466-y

(c) Der/die Autor(en) 2021

T. Teismann · A. Gysin-Maillart

\section{Psychotherapie nach einem Suizidversuch - Evidenzlage und Bewertung}

\section{Zusammenfassung}

Suizidversuche gelten als einer der wichtigsten Risikofaktoren für Suizide. Vor diesem Hintergrund wurden in den letzten Jahren diverse Psychotherapieangebote für Personen nach einem Suizidversuch entwickelt und untersucht. Im Rahmen dieses Artikels wird der aktuelle Stand der Effektivitätsforschung zusammengefasst, es werden Beispiele für erfolgreiche suizidfokussierte Psychotherapieprogramme gegeben und der gegenwärtige Forschungs- und Wissensstand wird kritisch reflektiert. Die Ergebnisse von 2 aktuellen Cochrane-Reviews zur Psychotherapie nach selbstverletzendem Verhalten im Kindes-, Jugend- und Erwachsenenalter sowie Befunde aus 14 weiteren Metaanalysen zur psychologischen Suizidprävention, die in

den vergangenen 5 Jahren publiziert wurden, werden überblicksartig dargestellt. Die kognitive Verhaltenstherapie (KVT) und die dialektisch-behaviorale Therapie (DBT) haben sich als effektiv erwiesen. Insgesamt sind die gemittelten Effektstärken jedoch von geringer Größe und diverse methodische Probleme verunmöglichen weitreichende Schlussfolgerungen. Grundsätzlich kommt der suizidspezifischen Psychotherapie in der individuumszentrierten Suizidprävention besondere Bedeutung zu; die empirische Fundierung und Dissemination entsprechender Programme sind jedoch noch unzureichend.

Schlüsselwörter

Suizidversuch · Suizid · Suizidprävention · Metaanalysen · Kognitive Verhaltenstherapie

\section{Psychotherapy after a suicide attempt-current evidence and evaluation}

\section{Abstract}

Suicide attempts are considered one of the most important risk factors for suicide. Based on this, various psychotherapeutic treatments for persons after a suicide attempt have been developed and evaluated in recent years. In this article, the current state of efficacy research is outlined, examples of successful suicide-focused psychotherapies are presented, and the current state of research and knowledge is critically reflected upon. The results of two recent Cochrane reviews of psychotherapy following selfinjurious behavior in childhood, adolescence, and adulthood, as well as findings from 14 other meta-analyses of psychological suicide prevention published in the past five years, are presented.
Cognitive behavioral therapy and dialectical behavioral therapy have been shown to be effective. Overall, however, the averaged effect sizes are of small magnitude and various methodological problems make it impossible to draw far-reaching conclusions. In principle, suicide-specific psychotherapy is of particular importance in individualcentered suicide prevention; however, the empirical foundation and dissemination of appropriate programs are still insufficient.

\section{Keywords}

Suicide attempt - Suicide · Suicide prevention . Meta-analyses - Cognitive-behavioural therapy und Messzeitpunkt(en) - nicht in allen Metaanalysen präventive Effekte finden, (3) insbesondere Onlineangebote mit einer hohen Abbrecherquote konfrontiert sind, (4) für Kinder und Jugendliche nur wenige effektive Behandlungsangebote bereitstehen, (5) nicht in allen Studien zwischen Suizidversuchen und nichtsuizidaler Selbstverletzung differenziert wird und (6) die methodische
Qualität eines Großteils der Studien gering ist (57,58\%; [23]).

Insgesamt würde das Feld davon profitieren, wenn systematisch solche Therapieansätze in methodisch adäquaten (Replikations-)Studien überprüft werden würden, die sich in ersten Untersuchungen als besonders effektiv herausgestellt haben, die potenziell störungs- und altersübergreifend einsetzbar sowie in manualisierter Form 
(Einzelschritte der Therapie in einem Handbuch dargelegt) verfügbar sind.

\section{Beispiele für effektive Psychotherapieprogramme}

Vor diesem Hintergrund sollen im Folgenden verschiedene Formen der KVT skizziert werden: die kognitive Therapie für suizidale Patient*innen (KT-SP; [31]), die kurze kognitive Verhaltenstherapie zur Suizidprävention (KVT-SP; [32]) und das Attempted Suicide Short Intervention Program (ASSIP; [33]). Sämtliche dieser Behandlungsprogramme haben sich in ersten Studien als effektiv erwiesen [10] und entsprechen den oben genannten Kriterien.

\section{Kognitive Therapie und kurze kognitive Verhaltenstherapie suizidaler Patienten}

Die kognitive Therapie suizidaler Patienten (KT-SP) und die kurze kognitive Verhaltenstherapie zur Suizidprävention (KVT-SP) verstehen sich als Kurzzeittherapien (10 bis 12 Sitzungen), die in Ergänzung zu anderen Therapien eingesetzt werden können und die sich in 3 Therapiephasen untergliedern lassen: die Eingangsphase, die mittlere Therapiephase und die Abschlussphase.

In der Eingangsphase geht es darum, die suizidale Krise zu verstehen, Ansatzpunkte für die Behandlung zu identifizieren, eine Risikoabschätzung vorzunehmen und krisentherapeutische Strategien zu implementieren. Die Behandlung nimmt ihren Ausgangspunkt mit dem sogenannten narrativen Interview, bei dem Betroffene aufgefordert werden, die Geschehnisse, die zum Suizidversuch geführt haben, in ihren Worten zu erzählen (vgl. ASSIP Punkt 1, - Tab. 2). In einer sich anschließenden hochauflösenden Kognitionsanalyse werden die Abläufe unmittelbar um den Suizidversuch herum dann nochmals detailliert betrachtet. Ergänzend wird in der Eingangsphase eine Risikoabschätzung vorgenommen, es wird ein Notfallplan erstellt und es werden Gründe, die für das Weiterleben sprechen, herausgearbeitet und verschriftlicht. Zusätzlich wird eine sogenannte Hope Box erstellt, d.h., die
Patienten werden darin unterstützt, eine Schachtel mit Gegenständen zu füllen, die an die persönlichen Gründe weiterzuleben erinnern.

In der mittleren Therapiephase werden - auf der Basis der individuellen Fallkonzeption - solche KVT-Standardmethoden gewählt, die im individuellen Fall das größte Potenzial haben, einem weiteren Suizidversuch vorzubeugen. Im Einzelnen kann es also darum gehen, dass (1) kognitive Infragestellungsmethoden genutzt werden, um suizidbezogene Annahmen/Vorstellungen („Ich bin eine Last für andere“) zu modifizieren, (2) Problemlösetrainings verwendet werden, um dem Eindruck der Ausweglosigkeit entgegenzutreten, (3) soziale Ängste und Inkompetenzen angegangen werden, um die soziale Integration zu fördern, (4) Skills und Achtsamkeitsübungen vermittelt werden, um den Umgang mit emotionalem Schmerz zu erleichtern, und (5) Maßnahmen zur Verbesserung des Schlafs und der Entspannungsfähigkeit genutzt werden, um einer weiteren emotionalen Eskalation entgegenzuwirken. Alle diese Techniken und Strategien werden in gleicher oder vergleichbarer Form auch in der kognitiven Verhaltenstherapie nichtsuizidaler Patient ${ }^{*}$ innen eingesetzt - sie sind also wenig spezifisch. Der Unterschied in der Anwendung ergibt sich ausschließlich dadurch, dass die Methoden, ausgehend von einem genauen Verständnis, wie eine suizidale Krise bei einem Betroffenen „funktioniert“, passgenau darauf abzielen, erneuten suizidalen Krisen, Suizidversuchen und Suiziden vorzubeugen.

In der Abschlussphase wird eine sogenannte Rückfallpräventionsübung (Relapse Prevention Task) genutzt, um die erworbenen Fertigkeiten imaginativ zu erproben. Bei der Rückfallpräventionsintervention handelt es sich um eine Imaginationsübung, in der Patient*innen angeleitet werden, sich eine zurückliegende und eine antizipierte zukünftige suizidale Krise imaginativ zu vergegenwärtigen und den Einsatz von Bewältigungsstrategien in sensu (in der Vorstellung) zu erproben. Die Technik dient sowohl zur Konsolidierung der Therapieinhalte als auch zur Kontrolle, ob Patient ${ }^{*}$ innen in der Lage sind, die entwickelten Strategien - zumindest in der Vorstellung einzusetzen.

Die Effektivität des beschriebenen Vorgehens konnte in 2 unabhängigen Studien gezeigt werden: Brown et al. [34] verglichen die KT-SP mit einer TAUBedingung bei 120 Patient*innen, die kurz zuvor einen Suizidversuch unternommen hatten. Im Studienzeitraum von 18 Monaten wiesen die Patient ${ }^{*}$ innen der Behandlungsgruppe eine nahezu $50 \%$ niedrigere Suizidversuchsrate auf als die Patient ${ }^{*}$ innen der TAU-Bedingung (24\% KT vs. $42 \%$ TAU). In der Untersuchung von Rudd et al. [35] erhielten 152 suizidale Soldat*innen entweder die KVT-SP oder eine TAU-Behandlung. Im Studienzeitraum von 24 Monaten wiesen die Patient ${ }^{*}$ innen der Behandlungsgruppe eine nahezu $60 \%$ niedrigere Suizidversuchsrate auf als die Patient*innen der TAU-Bedingung ( $14 \%$ KVT vs. $40 \%$ TAU). Die Befunde unterstreichen sehr deutlich das Potenzial des Behandlungsansatzes. Spezifikationen des allgemeinen therapeutischen Vorgehens liegen für Jugendliche und alte Menschen [31] sowie den stationären Kontext [36] vor. Eine Adaptation für den deutschen Sprachraum steht bislang aus.

\section{Attempted Suicide Short Intervention Program (ASSIP)}

Eine kurztherapeutische Behandlungsalternative stellt das Attempted Suicide Short Intervention Program (ASSIP) von Gysin-Maillart [33] dar. Die Behandlung umfasst insgesamt 3-4 Sitzungen mit einem anhaltenden Kontaktangebot durch das Zusenden von Briefen. Die Behandlung zielt darauf $a b$, die Hintergründe einer suizidalen Krise zu klären und Strategien zur Prävention erneuter suizidaler Handlungen zu entwickeln. ASSIP wird dabei immer als Zusatzangebot zu einer regulär laufenden Behandlung (ambulant, teilstationär, stationär) angeboten (- Tab. 2).

Die Effektivität von ASSIP wurde bislang in einer randomisiert kontrollierten Studie gezeigt: Gysin-Maillart und Kolleg*innen [37] verglichen ASSIP plus TAU mit einer reinen TAU-Bedingung bei 120 Patient ${ }^{*}$ innen, die zuvor einen 
(1) In der ersten Sitzung, dem narrativen Interview, werden die Patient*innen gebeten, ihre Geschichte, die zum Suizidversuch geführt hat, zu erzählen („Ich möchte Sie bitten mir zu erzählen, wie es zu diesem Suizidversuch kam“). Den Patient*innen wird Raum gegeben, die Geschichte so zu erzählen, wie sie sie erlebt haben. Therapeut*innen sind angehalten, Pausen zuzulassen und die Erzählung nicht durch Fragen zu unterbrechen. Erst wenn Patient*innen mit ihrer Geschichte zum Ende kommen, sollten Therapeut*innen offene Verständnisfragen klären. Das Ziel des narrativen Interviews besteht darin, ein gemeinsames, patientenorientiertes Verständnis der suizidalen Krise in einem biografischen Kontext zu entwickeln. Im Mittelpunkt stehen die narrative Kompetenz von Patient*innen und der Aufbau einer frühen therapeutischen Beziehung. Die Sitzung wird auf Video aufgezeichnet. Psychoedukative Unterlagen (,Suizid ist keine überlegte Handlung“) werden den Patient ${ }^{*}$ innen mit nach Hause gegeben

(2) In der zweiten Sitzung wird der Hausaufgabentext besprochen und persönliche Erfahrungen mit den wichtigen theoretischen Konzepten in Zusammenhang gebracht. Im Anschluss wird ein Video-Playback durchgeführt, in dem Patient*in und Therapeut*in Seite an Seite sitzen und ausgewählte Sequenzen des aufgezeichneten Narrativs anschauen. Dabei werden individuelle Muster, die zu suizidalem Verhalten führten, sowie persönliche Vulnerabilitäten und Trigger-Ereignisse identifiziert. Ziel des Video-Playbacks ist zudem, den mentalen Zustand (den sogenannten suizidalen Modus) der Patient*innen in einem sicheren Setting zu reaktivieren und gleichzeitig den Übergang des erlebten psychischen Schmerzes/Stresses hin zu suizidalem Verhalten Schritt für Schritt zu analysieren. Automatische Gedanken, Gefühle, physiologische Veränderungen und daraus resultierende Verhaltensmuster werden identifiziert und präventive Verhaltensmaßnahmen erarbeitet. Zugleich werden eine kognitive Umstrukturierung und eine emotionale Neuintegration ermöglicht. Für die dritte Sitzung bereiten die Therapeut*innen eine schriftliche Zusammenfassung der Abläufe der suizidalen Krise vor

(3) In der dritten Sitzung wird dieser schriftliche Entwurf der individuellen und suizidspezifischen Fallkonzeption gemeinsam überarbeitet. Muster, Abläufe und längerfristige Therapieziele, die im Zusammenhang mit suizidalen Krisen stehen, aber auch persönliche Warnzeichen und suizidspezifische Bewältigungsstrategien werden erarbeitet und in der Fallkonzeption suizidalen Verhaltens festgehalten. Anschließend werden diese - im Sinne eines Notfallplans - auf einen kreditkartengroßen Leporello (Zick-Zack-Faltheft) kopiert. Zusätzlich erhalten die Patient*innen eine Notfallkarte mit wichtigen Notfallnummern, um einen einfachen Zugang zum Helfersystem zu garantieren

(4) In der vierten Sitzung, die optional angeboten wird, werden die erarbeiteten Strategien im Rahmen einer erneuten Videoexposition eingeübt: Die Patient*innen betrachten die Videoaufnahme aus der ersten Sitzung und werden aufgefordert, die Geschichte ihres suizidalen Erlebens und Verhaltens mit den erarbeiteten Strategien probeweise zu unterbrechen. Im Anschluss an die Sitzungen bekommen die Patient*innen über 2 Jahre hinweg regelmäßig halbstandardisierte Briefe zugeschickt. Hierbei werden die Patient*innen daran erinnert, dass auch in Zukunft suizidale Krisen auftreten können und sie dabei ihre eigenen erarbeiteten Strategien nutzen sollen. Diese Vorgehensweise dient dem Ziel, eine lockere, aber verbindliche therapeutische Verankerung zu halten und einen einfachen Zugang zum Helfersystem zu gewährleisten

Suizidversuch unternommen hatten. Im Studienzeitraum von 24 Monaten kam es zu 5 Suizidversuchen in der ASSIPBedingung und $\mathrm{zu} 41$ Suizidversuchen in der Kontrollbedingung. Der Anteil an Patient ${ }^{\star}$ innen, die einen oder mehrere Suizidversuche unternahmen, lag bei $8,3 \%(n=5)$ in der ASSIP-Bedingung, im Vergleich zu 26,7\% $(n=16)$ in der Kontrollbedingung. Patient ${ }^{*}$ innen der ASSIP-Gruppe hatten ein annähernd $80 \%$ geringeres Risiko, einen späteren Suizidversuch zu unternehmen, als $\mathrm{Pa}$ tient ${ }^{*}$ innen der TAU-Bedingung.

Die Ergebnisse verweisen auf den präventiven Nutzen von ASSIP. Allerdings bleiben die Ergebnisse verschiedener - derzeit laufender - Replikationsstudien und die damit verbundenen klinischen Implikationen abzuwarten.

\section{Diskussion}

Die beschriebenen Interventionsbeispiele verweisen auf die Möglichkeit effektiver psychotherapeutischer Suizidprävention und empfehlen sich für eine fortgesetzte empirische Überprüfung.
Gleichwohl behalten die im Rahmen metaanalytischer Untersuchungen sichtbar gewordenen Probleme ihre Gültigkeit: Die gemittelten Effektstärken sind gering (und dies in Bezug auf sämtliche suizidalitätsbezogene Outcome-Maße und im Vergleich zu TAUBedingungen $)^{1}$, die methodische Qualität eines Großteils der Studien ist unzureichend und es fehlt nahezu vollständig an Replikationsstudien. Insbesondere Letzteres ist hierbei kritisch zu werten. So gibt es - vor allem aus dem Bereich der psychotherapeutischen Suizidprävention im Kindes- und Jugendalter verschiedene Beispiele, in denen initial vielversprechende Behandlungsergebnisse [38-40] in Folgestudien nicht repliziert werden konnten [41-43].

Zudem wurden nur in Ausnahmefällen verschiedene aktive Behandlungsformen gegeneinander getestet, sodass sich zur Über- oder Unterlegenheit verschiedener Psychotherapieverfahren so-

\footnotetext{
1 Es sei allerdings angemerkt, dass TAU alleine nicht selten einen deutlich positiven Effekt zeigt [24].
}

wie $\mathrm{zu}$ differenziellen Indikationen bislang keine Aussagen treffen lassen. Es fehlt an Studien zur Effektivität suizidfokussierter Psychotherapieverfahren im Kontext unterschiedlicher Störungsbilder (u.a. Essstörungen), Settings (u.a. stationäre Psychotherapie) und im Rahmen unterschiedlicher Gesundheitssysteme (z. B. Deutschland, Schweiz, USA). Schlussendlich fehlt es an ausreichend gepowerten multizentrischen Studien, in denen die Prävention von Suiziden, anstatt der Prävention von Suizidversuchen und/oder Suizidgedanken als OutcomeMaß gewählt wurde.

Trotz aller berechtigten Kritik scheint die suizidfokussierte Psychotherapie die derzeit aussichtsreichste Kandidatin unter den individuumszentrierten Interventionen zu sein: Mit Ausnahme von Lithium [44] konnte bislang für kein Medikament eine stabile antisuizidale Wirkung nachgewiesen werden [23] und andere somatische Behandlungsverfahren (Elektrokrampftherapie, Schlafentzug) wurden zu selten untersucht, um eine Einschätzung vornehmen zu können. Vor dem Hintergrund der 
Ergebnisse der Cochrane-Reviews von Witt und Kolleg*innen [6, 7] empfehlen sich insbesondere KVT, DBT und MBT für weitere Wirksamkeitsstudien.

\section{Fazit}

Es lässt sich festhalten, dass spezifische psychotherapeutische Angebote für Patient*innen nach einem Suizidversuch einen wichtigen Beitrag zur Suizidprävention leisten können. Die empirische Fundierung und Dissemination spezifischer suizidfokussierter Psychotherapie ist jedoch unzureichend und es bedarf strategischer und konzertierter Forschungsbemühungen, um hier Fortschritte zu erzielen.

\section{Korrespondenzadresse}

\section{PD Dr. Tobias Teismann}

Forschungs- und Behandlungszentrum für Psychische Gesundheit, Fakultät für Psychologie, Ruhr-Universität Bochum Massenbergstr. 11, 44788 Bochum, Deutschland tobias.teismann@rub.de

Funding. Open Access funding enabled and organized by Projekt DEAL.

\section{Einhaltung ethischer Richtlinien}

Interessenkonflikt. T. Teismann und A.Gysin-Maillart geben an, dass kein Interessenkonflikt besteht.

Für diesen Beitrag wurden von den Autoren keine Studien an Menschen oder Tieren durchgeführt. Für die aufgeführten Studien gelten die jeweils dort angegebenen ethischen Richtlinien.

Open Access. Dieser Artikel wird unter der Creative Commons Namensnennung 4.0 International Lizenz veröffentlicht, welche die Nutzung, Vervielfältigung, Bearbeitung, Verbreitung und Wiedergabe in jeglichem Medium und Format erlaubt, sofern Sie den/die ursprünglichen Autor(en) und die Quelle ordnungsgemäß nennen, einen Link zur Creative Commons Lizenz beifügen und angeben, ob Änderungen vorgenommen wurden.

Die in diesem Artikel enthaltenen Bilder und sonstiges Drittmaterial unterliegen ebenfalls der genannten Creative Commons Lizenz, sofern sich aus der Abbildungslegende nichts anderes ergibt. Sofern das betreffende Material nicht unter der genannten Creative Commons Lizenz steht und die betreffende Handlung nicht nach gesetzlichen Vorschriften erlaubt ist, ist für die oben aufgeführten Weiterverwendungen des Materials die Einwilligung des jeweiligen Rechteinhabers einzuholen.
Weitere Details zur Lizenz entnehmen Sie bitte der Lizenzinformation auf http://creativecommons.org/ licenses/by/4.0/deed.de.

\section{Literatur}

1. Statistisches Bundesamt (2021) Todesursachenstatistik. www.destatis.de, Zugegriffen: April 2021.

2. Nock MK, Borges G, Ono Y (2014) Suicide. Global perspectives from the WHO World Mental Health Survey. Cambridge University Press, Cambridge

3. Donath C, Graessel E, Baier D, Bleich S, Hillemacher T (2014) Is parenting style a predictor of suicide attempts in a representative sample of adolescents? BMC Pediatr 14:113-125. https://doi. org/10.1186/1471-2431-14-113

4. WHO (2014) Preventing suicide. A global imperative. WHO Press, Genf.

5. Caroll R, Metcalfe C, Gunnell D (2014) Hospital presenting self-harm and risk of fatal and non-fatal repetition. PLOSOne 9:e89944. https://doi.org/10. 1371/journal.pone.0089944

6. Witt KG, Hetrick SE, Rajaram G, Hazell P, Salisbury TTL, Townsend E, Hawton K (2021) Psychosocial interventions for self-harm in adults. Cochrane Database Syst Rev. https://doi.org/10.1002/ 14651858.CD013668.pub2

7. Witt KG, Hetrick SE, Rajaram G, Hazell P, Salisbury TTL, Townsend E, Hawton K (2021) Interventions for self-harm in children and adolescents. Cochrane Database Syst Rev. https://doi.org/10.1002/ 14651858.CD013667.pub2

8. Hetrick SE, Robinson J, Spittal MJ, Carter G (2016) Effective psychological and psychosocial approaches to reduce repetition of self-harm: a systematic review, meta-analysis and metaregression. Bmj Open 6:e11024. https://doi.org/ 10.1136/bmjopen-2016-011024

9. Kapur N, Cooper J, O'Connor RC, Hawton K (2013) Non-suicidal self-injury v. attempted suicide: new diagnosis or false dichotomy? Br J Psychiatry 202:326-328. https://doi.org/10.1192/bjp.bp.112. 116111

10. Gøtzsche PC, Gøtzsche PK (2017) Cognitive behavioural therapy halves the risk of repeated suicide attempts: systematic review. J R Soc Med 110: 404-410. https://doi.org/10.1177/ 0141076817731904

11. Hawton K, Witt KG, Salisbury TTL, Arensman E, Gunnell D, Hazell P, Townsend E, van Heeringen $K$ (2016) Psychosocial interventions for self-harm in adults. Cochrane Database Syst Rev 5:CD12189. https://doi.org/10.1002/14651858.CD012189

12. Hofstra $E$, Van Nieuwenhuizen $C$, Bakker $M$, Özgül D, Elfeddali I, de Jong SJ, van der FeltzCornelis CM (2020) Effectiveness of suicide prevention interventions: a systematic review and meta-analysis. Gen Hosp Psychiatry 63:127-140. https://doi.org/10.1016/j.genhosppsych.2019.04. 011

13. Robinson J, Bailey E, Witt K, Stefanac N, Milner A, Currier D, Pirkis J, Condron P, Hetrick S (2018) What works in youth suicide prevention? A systematic review and meta-analysis. EClinicalMedicine 4:52-91. https://doi.org/10.1016/j.eclinm.2018. 10.004

14. Nuij $C$, van Ballegooijen W, de Beurs D, Juniar D, Erlangsen A, Portzky G, O'Connor RC, Smit JH, Kerkhof A, Riper H (2021) Safety planning type interventions for suicide prevention: meta- analysis. Br J Psychiaty. https://doi.org/10.1192/ bjp.2021.50.

15. Doupnik SK, Rudd B, Schmutte T, Worsley D, Bowden CF, McCarthy E, Eggan E, Bridge JA, Marcus SC (2020) Association of suicide prevention interventions with subsequent suicide attempts, linkage to follow-up care, and depression symptoms for acute care settings: a systematic review and metaanalysis. JAMA Psychiatry 77 : 1021-1030. https:// doi.org/10.1001/jamapsychiatry.2020.1586.

16. Witt K, de Moraes DP, Salisbury TT, Arensman E, Gunnell D, Hazell P, Townsend E, van Heeringen K, Hawton K (2018) Treatment as usual (TAU) as a control condition in trials of cognitive behavioural-based psychotherapy for self-harm: Impact of content and quality on outcomes in a systematic review. J Affect Disord 235:434-447. https://doi.org/10.1016/j.jad.2018.04.025

17. Yuan SNV, Kwok KHR, Ougrin D (2019) Treatment engagement in specific psychological treatment vs. treatment as usual for adolescents with selfharm: systematic review and meta-analysis. Front Psychol 10:104. https://doi.org/10.3389/fpsyg. 2019.00104

18. Bornheimer LA, Zhang A, Li J, Hiller M, Tarrier N (2020) Effectiveness of suicide-focused psychosocial interventions in psychosis: a systematic review and meta-analysis. Psychiatr Serv 71:829-838. https://doi.org/10.1176/appi.ps.201900487

19. Briggs S, Netuveli G, Gould N, Gkaravella A, Gluckman NS, Kangogyere P, Farr R, Goldblatt MJ, Lindner R (2019) The effectiveness of psychoanalytic/psychodynamic psychotherapy for reducing suicide attempts and self-harm: systematic review and meta-analysis. Br J Psychiatry 214:320-328. https://doi.org/10.1192/bjp.2019.33

20. BüscherR, TorokM, TerhorstY,SanderL(2020) Internet-based cognitive behavioral therapy to reduce suicidal ideation: a systematic review and metaanalysis. JAMA Netw Open 3: e203933. https://doi. org/10.1001/jamanetworkopen.2020.3933.

21. CalatiR, CourtetP(2016) Is psychotherapy effective for reducing suicide attempt and nonsuicidal selfinjury rates? Meta-analysis and meta-regression of literature data. J Psychiatr Res 79:8-20. https://doi. org/10.1016/j.jpsychires.2016.04.003

22. DeCou CR, Comtois KA, Landes SJ (2019) Dialectical behavior therapy is effective for the treatment of suicidal behavior: a meta-analysis. Behav Ther 50:60-72.https://doi.org/10.1016/j.beth.2018.03. 009

23. Fox KR, Huang $X$, Guzmán EM, Funsch KM, Cha CB, Ribeiro JD, Franklin JC (2020) Interventions for suicide and self-injury: A meta-analysis of randomized controlled trials across nearly 50 years of research. Psychol Bull 146:1117-1145. https:// doi.org/10.1037/bul0000305

24. Kothgassner OD, Robinson K, Goreis A, Ougrin D, Plener PL (2020) Does treatment method matter? A meta-analysis of the past 20 years of research on therapeutic interventions for self-harm and suicidal ideation in adolescents. Borderline Personal Disord Emot Dysregul 7:9. https://doi. org/10.1186/s40479-020-00123-9

25. LeaveyK, Hawkins R(2017) Is cognitive behavioural therapy effective in reducing suicidal ideation and behaviour when delivered face-to-face or via e-health? A systematic review and meta-analysis. Cogn Behav Ther 46:353-374. https://doi.org/10. 1080/16506073.2017.1332095

26. Meerwijk EL, Parekh A, Oquendo MA, Allen IE, Franck LS, Lee KA (2016) Direct and indirect psychosocial and behavioral interventions to prevent suicide and suicide attempt: a systematic review 


\section{Leitthema}

and meta-analysis. Lancet Psychiatry 3: 544-554 https://doi.org/10.1016/S2215-0366(16)00064-X

27. Padmanathan $P$, Hall $K$, Moran $P$, Jones HE, Gunnell D, Carlisle V, Lingford-Hughes A, Hickman M (2020) Prevention of suicide and reduction of self-harm among people with substance use disorder: a systematic review and meta-analysis of randomised controlled trials. Compr Psychiatry 96:152135. https://doi.org/10.1016/j.comppsych. 2019.152135

28. Riblet NBV, Shiner B, Young-Xu Y, Watts BV (2017) Strategies to prevent death by suicide: metaanalysis of randomized controlled trials. $\mathrm{Br} \mathrm{J}$ Psychiatry 210:396-402. https://doi.org/10.1192/ bjp.bp. 116.187799

29. Swift JK, Trusty WT, Penix EA (2021) The effectiveness of the Collaborative Assessment and Management of Suicidality (CAMS) compared to alternative treatment conditions: A meta-analysis. Suicide Life Threat Behav. https://doi.org/10.1111/ sltb. 12765

30. Torok M, Han J, Baker S, Werner-Seidler A, Wong I, Larsen ME, Christensen H. (2020) Suicide prevention using self-guided digital interventions: a systematic review and metaanalysis of randomised controlled trials. Lancet Digital Health 2: 25-36. https://doi.org/10.1016/ S2589-7500(19)30199-2

31. Wenzel A, Brown G, Beck AT (2009) Cognitive therapy for suicidal patients: scientific and clinical applications. American Psychological Association, Washington

32. Bryan CJ, Rudd MD (2018) Brief cognitivebehavioral therapyfor suicide prevention. Guilford, New York

33. Gysin-Maillart A (2021) ASSIP - Kurztherapie nach Suizidversuch. Attempted Sucide Short Intervention Program, 2. Aufl. Hogrefe, Bern

34. Brown GK, TenHave T, Henriques G, Xie S, Hollander J, Beck AT (2005) Cognitive therapy for the prevention of suicide attempts. a randomized controlled trial. JAMA 294: 563-570. https://doi. org/10.1001/jama.294.5.563

35. Rudd MD, Bryan CJ, Wertenberger EG, Peterson AL, Young-McCaughan S, Mintz J, WilliamsSR, ArneKA, Breitbach J, Delano K, Wilkinson E, Bruce TO (2015) Brief cognitive-behavioral therapy effects on posttreatment suicide attempts in a military sample: results of a randomized clinical trial with 2-year follow up. Am J Psychiatry 172:441-449. https:// doi.org/10.1176/appi.ajp.2014.14070843

36. Ghahramanlou-Holloway M, LaCroix JM, Perera KU, Neely L, Grammer G, Weaver J, Novak LA, Colborn V, Lee-Tauler SY (2020) Inpatient psychiatric care following a suicide-related hospitalization: A pilot trial of Post-Admission Cognitive Therapy in a military medical center. Gen Hosp Psychiatry 63:46-53. https://doi.org/10.1016/j.genhosppsych.2018.11. 006

37. Gysin-Maillart A, Schwab S, Soravia L, Megert M, Michel K (2016) A novel brief therapy for attempted suicide: Two-year follow-up randomized controlled study of the Attempted Suicide Short Intervention Program (ASSIP). PLoS Med 13:e1001968. https://doi.org/10.1371/journal. pmed.1001968

38. Diamond GS, Wintersteen M, Brown G, Gallop R (2010) Attachment-based family therapy for adolescents with suicidal ideation: a randomized controlled trial. J Am Acad Child Adolesc Psychiatry 49:122-131. https://doi.org/10.1097/00004583201002000-00006

39. Evans K, Tyrer P, Catalan J, Schmidt U, Davidson K, Dent J, Tata P, Thornton S, Barber J, Thompson S
(1999) Manual-assisted cognitive-behaviour therapy (MACT): a randomized controlled trial of a brief intervention with bibliotherapy in the treatment of recurrent deliberate self-harm. Psychol Med 29:19-25. https://doi.org/10.1017/ s003329179800765x

40. Wood A, Trainor G, Rothwell J, Moore A, Harrington $R$ (2001) Randomized trial of group therapy for repeated deliberate self-harm in adolescents. J Am Acad Child Adolesc Psychiatry 40:1246-1253. https://doi.org/10.1097/00004583-20011100000003

41. Diamond GS, Kobak RR, Krauthamer Ewing ES, Levy SA, Herres JL, Russon JM, Gallop RJ (2019) A randomized controlled trial: attachment-based family and nondirective supportive treatments for youth who are suicidal. J Am Acad Child Adolesc Psychiatry 58:721-731. https://doi.org/10.1016/j. jaac.2018.10.006

42. Green JM, Wood AJ, Kerfoot MJ, Trainor G, Roberts C, Rothwell J, Woodham A, Ayodeji E, Barrett B, Byford S, Harrington R (2011) Group therapy for adolescents with repeated self harm: randomised controlled trial with economic evaluation. BMJ 342:d682. https://doi.org/10. 1136/bmj.d682

43. Tyrer $\mathrm{P}$, Thompson S, Schmidt U, Jones V, Knapp M Davidson K et al (2003) Randomized controlled trial of brief cognitive behavior therapy versus treatment as usual in recurrent deliberate selfharm. Psychol Med 33:969-976. https://doi.org/ 10.1017/s0033291703008171

44. Lorenzen T, Bschor T (2021) Medikamentöse Behandlung suizidalen Erlebens und Verhaltens. In: Teismann T, Forkmann T, Glaesmer H (Hrsg) Suizidales Erleben und Verhalten. Ein Handbuch. Psychiatrie-Verlag, Köln, S174-187 